\title{
Nodulation in vitro d'Acacia mangium Willd (Leguminosae)
}

\author{
A Galiana ${ }^{*}$, J Alabarce 1, E Duhoux ${ }^{2}$ \\ 1 CTFT/ORSTOM, biotechnologie des systèmes symbiotiques forestiers tropicaux; \\ 2 CTFT/ORSTOM, université Paris VII et biotechnologie des systemes symbiotiques \\ forestiers tropicaux, 45 bis, avenue de la Belle Gabrielle, 94736 Nogent-sur-Marne Cedex, France
}

(Reçu le 13 février 1990; accepté le 10 avril 1990)

\begin{abstract}
Résumé - Un nouveau dispositif de culture in vitro a permis d'étudier les conditions de la nodulation de jeunes plantes d'Acacia mangium inoculées avec la souche de Bradyrhizobium sp Aust13c. La cinétique de la nodulation a été suivie pendant plus de 3 mois et a permis de mettre en évidence 3 vagues successives de formation de nodules. La répartition des nodules le long du système racinaire, l'activité réductrice de l'acétylène et la croissance des plantes nodulées ont été étudiées en fonction de l'áge de la plante au moment de l'inoculation et de la présence ou non d'une source d'azote minéral ( $\left.\mathrm{KNO}_{3}, 0,2 \mathrm{mmol} / \mathrm{l}\right)$. Le dispositif utilisé a permis d'optimiser les conditions de la nodulation in vitro et a facilité les observations de la nodulation au cours de la croissance des plantes. Ce dispositif expérimental est désormais utilisé pour sélectionner de manière précoce les 2 partenaires de la symbiose Acacia mangium - Bradyrhizobium sp et pourrait être adapté à l'étude de la nodulation d'autres Légumineuses ligneuses.
\end{abstract}

nodulation In vitro / Acacia mangium / Bradyrhizobium sp / fixation biologique de l'azote

Summary - In vitro nodulation In young plants of Acacla mangium Willd (Leguminosae). $A$ new in vitro method was developed to compare the effect of early versus late inoculation on nodulation of Acacia mangium seedlings by Bradyrhizobium sp strain Aust $13 \mathrm{c}$. During the 3-month culture period, the time course of nodulation exhibited 3 active phases separated by 2 stationary phases (the first one $10 d$, the second $45 d$-long).

When plants were inoculated early (ie immediately, $T(I)=0 d$ after germination or $T(15)=15 d$ after germination) in the absence of combined $N$, nodulation was restricted to the basal part of the root system and nodule number was the highest. in the presence of combined nitrogen (KNO ${ }_{3}, 0.2$ mmol/i) nodulation shifted slightly towards the root apex.

When plants were inoculated later $(T(I)=30 d$ after germination) in the absence of combined nitrogen, nodulation occurred at the apex of the root system and nodule number was the highest when nitrogen was added to the culture medium.

After 5 months of growth, nodule number and dry weight, shoot dry weight and acetylene reduction activity (ARA) were significantly higher $(\mathrm{P}=0.05$ ) in plants inoculated early (immediately or $15 d$ after germination) than in plants inoculated later. Application of combined nitrogen to the medium did not significantly $(P=0.05)$ affect shoot dry weight, nodulation and ARA.

The method used proved to be the most convenient to study nodulation during plant growth because it allowed continuous and non destructive observations to be made. In the future, it could probably be used for early selection of the 2 partners of the Acacia mangium-Bradyrhizobium $\mathrm{sp}$ symbiosis and would also be suitable in nodulation studies of other woody legumes.

in vitro nodulation / Acacia mangium / Bradyrhizobium sp / nitrogen fixation

* Correspondance et tirés à part 


\section{INTRODUCTION}

Acacia mangium est un arbre fixateur d'azote originaire d'Australie. II est introduit en plantations industrielles dans les zones tropicales humides depuis plusieurs années, notamment en Asie du Sud-Est, pour son bois à usages multiples et pour sa croissance très rapide avec en moyenne $20 \mathrm{~m}^{3} \cdot \mathrm{ha}^{-1} \cdot \mathrm{an}^{-1}$ (Nat Acad Sci USA, 1983). L'aptitude d'A mangium à croître sur des sols dégradés et acides particulièrement déficients en azote, grâce notamment à son aptitude à fixer l'azote atmosphérique (NFT Highlights, 1987), en fait une espèce qui pourrait être utilisée dans les systèmes agroforestiers ou dans le cadre de la restauration de sols appauvris par des cultures intensives (Pomier et al, 1986).

Pour une plante hôte donnée, la fixation biologique de l'azote dépend de l'infectivité et de l'effectivité des souches de rhizobium utilisées. L'infectivité d'une souche de rhizobium est caractérisée par la distribution et le nombre de nodules formés sur l'ensemble de l'appareil racinaire des plants inoculés. Pour réaliser une étude expérimentale de la nodulation, différents dispositifs ont été utilisés dans les conditions de laboratoire, soit en conditions semi-aseptiques : sachets de croissance (growth pouches) (Bhuvaneswari et al, 1980, 1981; Turgeon et Bauer, 1982; Takats, 1986), surfaces gélosées (Dart, 1977), soit totalement aseptiques : géloses aérées (Lakshminarayana et al, 1988), sable (Dhawan et Bhojwani, 1987). Nous avons procédé pour notre étude à l'inoculation de jeunes plantes ${ }^{*} A$ mangium dans un dispositif in vitro amélioré où la plante est en asepsie complète et les nodules situés hors du milieu de culture. D'une manière générale, les premières étapes de la croissance des arbres sont plus lentes que celles des plantes annuelles. Notre dispositif in vitro semble donc particulièrement adapté aux plantes ligneuses fixatrices d'azote qui peuvent, comme A mangium, être cultivées pendant plus de 6 mois dans les tubes de culture.

La présente étude avait pour but d'examiner l'effet de la date d'inoculation après la germination des graines, en présence ou en l'absence d'une faible dose d'azote minéral, sur la distribution des nodules le long de l'appareil racinaire ainsi que sur la croissance et l'activité fixatrice de $\mathrm{N}_{2}$ des plantes. Elle a permis également de suivre avec précision la cinétique de nodulation au cours des $106 \mathrm{j}$ qui ont suivi l'inoculation.

\section{MATÉRIEL ET MÉTHODES}

\section{Plante hôte}

Les graines d'A mangium Willd (tribu des Phyllodineae) ont été récoltées en Australie (provenance Rex Range Queensland, référence CTFT $84 / 4136$ N).

\section{Bradyrhizobium}

La souche de Bradyrhizobium sp Aust13c, isolée au laboratoire à partir de nodules d'A mangium récoltés en Australie (Galiana et al, 1990), a été inoculée aux plantes après $7 \mathrm{j}$ de culture à $28^{\circ} \mathrm{C}$ sur milieu YEM (Vincent, 1970).

\section{Tubes de culture}

Le système de culture est constitué par un tube en verre $(25 \mathrm{~mm} \times 150 \mathrm{~mm})$ dans lequel ont été introduites 2 mottes Milcap ${ }^{1}$ superposées main-

1 MILCAP, Le Courtil, Chemin de Montbault Nuaillé 49340 Trémentines 
tenues par une baguette de verre au-dessus d'un réservoir de solution nutritive (fig 1). Ces mottes sont constituées de fibres de polypropylène chimiquement inertes, très aérées mais qui retiennent mal l'eau. Une mèche latérale de papier filtre qui plonge dans le réservoir en assure l'imbibition. Les plantes sont alimentées avec un milieu minéral de base sans azote - N (Anonyme, 1983) ou avec le même milieu enrichi en azote $+\mathrm{N}\left(\mathrm{KNO}_{3}, 0,2 \mathrm{mmol} / \mathrm{l}\right)$. Le milieu de base est dilué 4 fois afin d'éviter une concentration excessive des sels minéraux lors de l'évaporation de l'eau. Cette faible concentration en azote qui assure un bon début de croissance à la plante est couramment utilisée sous le nom d'azote starter (Rigaud, 1981). Chaque tube reçoit $20 \mathrm{ml}$ de milieu nutritif au moment de la mise en place de la plante dans le tube. Le milieu de culture est renouvelé tous les 3 mois. Le tube est obturé par un bouchon en ouate de cellulose. Les tubes de culture sont stérilisés à l'autoclave $\left(20 \mathrm{~min}\right.$ à $\left.120^{\circ} \mathrm{C}\right)$.

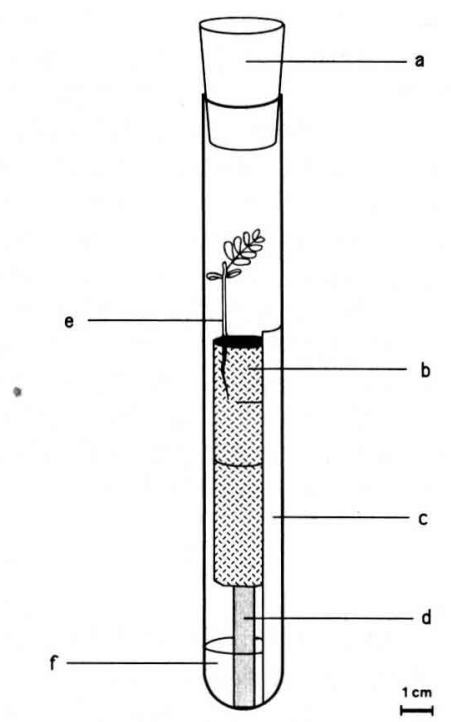

Fig 1. Schéma d'un tube de culture. a, bouchon en ouate de cellulose; b, support Milcap en fibres de polypropylène; c, mèche en papier filtre; $d$, tige-support en verre; e, plante reposant sur le bord extérieur du Milcap; $f$, solution nutritive.

\section{Mise en place et inoculation des plantes}

Les graines sont scarifiées à l'acide sulfurique concentré $(95 \%)$ pendant $1 \mathrm{~h}$, puis désinfectées dans une solution à $5 \%$ d'hypochlorite de calcium $(70 \%$ de chlore actif) pendant $20 \mathrm{~min}$. Après 3 rinçages de $10 \mathrm{~min}$ à l'eau distillée stérile, les graines sont mises à germer à la lumière sur eau gélosée en boîte de Petri. Huit jours après la scarification des graines, les jeunes plantes sont transférées dans les tubes de culture et placées à la surface de la motte Milcap. La jeune racine est alors disposée entre la motte et la paroi du tube de culture, ce qui permet l'observation continue de la nodulation (fig 2).

L'inoculation est réalisée en déposant, à l'aide d'une seringue, $1 \mathrm{ml}$ de culture pure liquide de Bradyrhizobium sp (souche Aust13C) de $7 \mathrm{j}$, contenant $10^{9}$ bactéries $/ \mathrm{ml}$, au contact des racines de la plante. L'appareil racinaire est

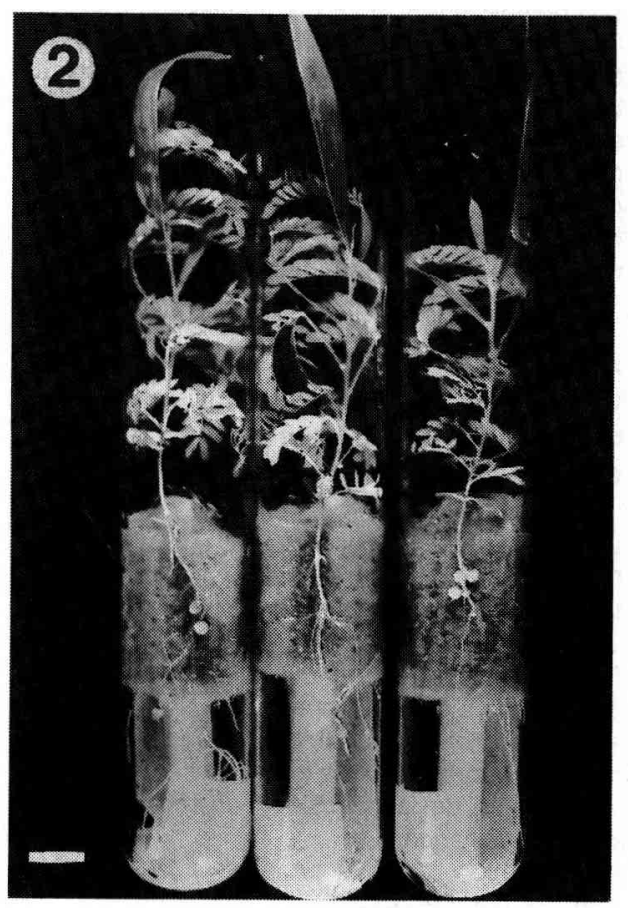

Fig 2. Jeunes plantes d'Acacia mangium âgées de 5 mois nodulées in vitro dans les tubes de culture (la barre horizontale représente $1 \mathrm{~cm}$ ). 
protégé de la lumière par un manchon en plastique noir amovible qui recouvre la partie inférieure du tube. Les tubes sont placés en chambre de culture à $27 \pm 1{ }^{\circ} \mathrm{C}$ avec une photopériode journalière de $16 \mathrm{~h}$ et sous une intensité lumineuse de $50 \mu \mathrm{E} \cdot \mathrm{m}^{-2} \cdot \mathrm{s}^{-1}$, en alternance avec $8 \mathrm{~h}$ d'obscurité à la température de $25 \pm$ $1{ }^{\circ} \mathrm{C}$.

\section{Observations et expression des résultats}

\section{Expérience préliminaire 1 : cinétique de nodulation et activité réductrice d'acétylène des plantes cultivées en tubes}

Une semaine après la scarification, les graines germées ont été inoculées au moment de leur transfert dans les tubes. A cette date $T(I)=0 \mathrm{j}$ on considère que les plantes sont âgées de $0 \mathrm{j}$. Les plantes ont ensuite été prélevées après 15, $28,35,70$ et $106 \mathrm{j}$ de croissance dans les tubes. Le nombre de nodules, le poids de matière sèche des nodules mesuré après sèchage à l'étuve pendant $72 \mathrm{~h}$, l'activité réductrice d'acétylène (ARA) mesurée par chromatographie en phase gazeuse selon les procédures habituelles (Hardy et al, 1968), ont été déterminés sur 10 plantes à chaque prélèvement.

\section{Expérience 2 : Etude de la nodulation dans le cas de plantes inoculées à différents âges en présence ou non d'azote starter}

Les plantes ont été cultivées sur milieu $+\mathrm{N}$ ou $\mathrm{N}$ et inoculées immédiatement $(T(I)=0 \mathrm{j})$ après leur mise en place dans les tubes de culture ou 15,30 et $45 \mathrm{j}$ plus tard $(T(I)=15,30$ ou $45 \mathrm{j})$. Dans ces différentes conditions, 3 types d'observations ont été effectuées :

- Distribution des nodules sur l'appareil racinaire $15 \mathrm{j}$ après l'inoculation;

- Cinétique de la nodulation en fonction de la date d'inoculation $T(1)$;

- Nodulation, $A R A$ et croissance des plantes après 5 mois de culture.

Dix plantes ont été observées pour chaque date d'inoculation et sur chaque milieu minéral avec ou sans azote.

\section{RÉSULTATS}

\section{Cinétique de la nodulation et de I'ARA sur des plantes inoculées à $0 \mathrm{j}(\mathrm{T}(\mathrm{I})=$ oj)}

Lorsque l'inoculation des plantes avec la souche de Bradyrhizobium sp Aust13c a lieu au moment de leur installation dans les tubes, soit à $T(I)=0 \mathrm{j}, 100 \%$ des plantes sont nodulées. La figure 3 montre que le nombre et le poids de matière sèche des nodules croissent tout au long de l'expérimentation, ce qui indique que les plantes sont encore physiologiquement actives après $106 \mathrm{j}$ dans ces conditions de culture. Dans notre dispositif, les nodules apparaissent et se développent environ 10-15 j après l'inoculation des plantes par la souche de Bradyrhizobium sp. Les nodules observés chez $\boldsymbol{A}$ mangium ont une morphologie qui évolue au cours du temps. Sphériques $(0,5$ à $1,5 \mathrm{~mm}$ de diamètre) quand ils sont jeunes, ils deviennent cylindriques $((2-4) \times 1,5 \mathrm{~mm}) 10 \mathrm{se}-$ maines après l'inoculation des plantes. Dans les conditions expérimentales adoptées, $A$ mangium présente 3 vagues successives de formation de nodules séparées par 2 périodes, la première de $10 \mathrm{j}$, la seconde de $45 \mathrm{j}$, pendant lesquelles aucun nodule ne se forme, alors que leur biomasse continue à croître de façon continue. L'ARA des plantes nodulées croît de façon exponentielle à partir du $28^{\mathrm{e}} \mathrm{j}$ de culture et commence à ralentir $70 \mathrm{j}$ après l'inoculation.

Influence de la date d'inoculation et de l'apport d'azote minéral sur la nodulation et I'ARA des plantes

\section{Distribution des nodules}

Le nombre et la distribution des nodules varient selon la date d'inoculation et en 


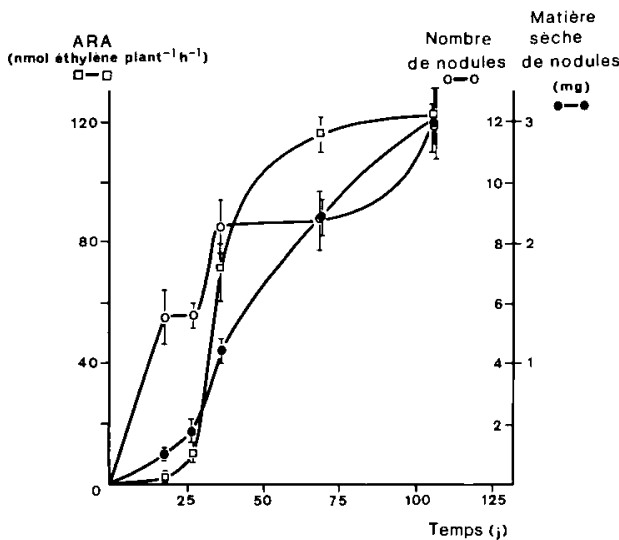

Fig 3. Cinétique de la nodulation et de l'activité réductrice d'acétylène chez Acacia mangium pendant 106 jours de culture. L'inoculation avec Bradyrhizobium sp (souche Aust13c) a eu lieu au moment du transfert des plantes dans les tubes de culture, soit au temps $T(I)=0$ jour (moyenne \pm erreur standard, $n=10$ ).

fonction de la présence ou de l'absence d'une source d'azote dans le milieu de culture. Les observations sont toujours effectuées $15 \mathrm{j}$ après l'inoculation (fig 4), et les dates d'observation $T(O)=15,30$ et $45 j$ correspondent donc respectivement aux dates d'inoculation $T(I)=0,15$ et $30 \mathrm{j}$.

La répartition des nodules varie en fonction de la date d'inoculation. En effet, une inoculation précoce à $T(I)=0 \mathrm{j}$ provoque une nodulation proche du collet de la plante tandis qu'une inoculation tardive à $T(I)=15$ et $30 \mathrm{j}$ provoque un étalement de la distribution des nodules le long de l'appareil racinaire. Chez les plantes inoculées aux temps $T(I)=0$ et $15 \mathrm{j}$, les nodules se situent au niveau des points d'émergence des racines secondaires, à proximité de la racine principale. Lorsque l'inoculation est plus tardive $(T(l)=30 \mathrm{j})$, les nodules se situent dans la région médiane des racines secondaires.

L'addition d'une faible dose de $\mathrm{KNO}_{3}$ $(0,2 \mathrm{mmol} / \mathrm{l})$ dans le milieu de culture provoque un léger déplacement de la distribu- tion des nodules du collet vers l'apex de la racine principale (fig 4 ) et a un effet positif sur le nombre de nodules formés à $T(I)=$ $30 \mathrm{j}$.

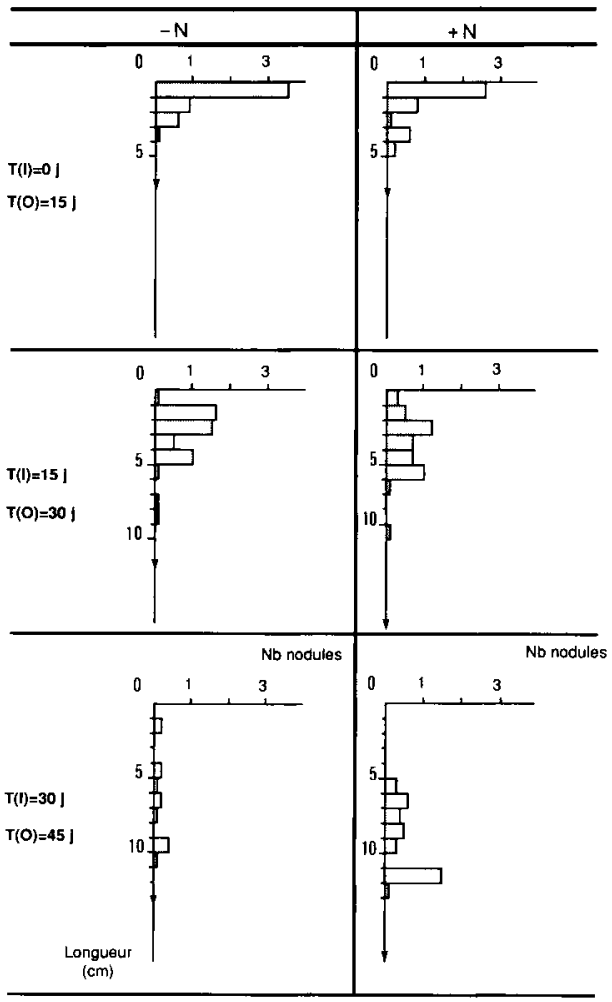

Fig 4. Répartition des nodules sur l'appareil racinaire d'A mangium en fonction de l'âge de la plante au moment de l'inoculation. $-\mathrm{N}$ : milieu minéral sans azote, $+\mathrm{N}$ : milieu minéral avec azote $\left(\mathrm{KNO}_{3}\right.$ 0,2 mmol/ $)$. $\mathrm{T}(\mathrm{l})$ : âge des plantes au moment de l'inoculation; $T(O)$ : âge des plantes au moment de l'observation, soit $15 \mathrm{j}$ après l'inoculation. La longueur moyenne de la racine principale est schématisée sur l'axe des ordonnées par une flèche qui représente son extrémité. L'appareil racinaire est subdivisé en sections de $1 \mathrm{~cm}$. Le nombre de nodules de chaque section est représenté par une barre horizontale. 


\section{Cinétique de la nodulation}

Sur milieu dépourvu de $\mathrm{KNO}_{3}$, le nombre de nodules formés pendant $60 \mathrm{j}$ se stabilise $15 \mathrm{j}$ après l'inoculation des plantes (fig 5). Le nombre maximal de nodules (67) est obtenu chez les plantes inoculées immédiatement $(T(I)=0 \mathrm{j})$ ou $15 \mathrm{j}(T(I)=$ 15 j) après la germination. Lorsque les plantes sont inoculées $30 \mathrm{j}(T(I)=30 \mathrm{j})$ ou $45 \mathrm{j}(T(I)=45 \mathrm{j})$ après la germination, 2 à 3 nodules par plante seulement sont formés.

Lorsque le milieu de culture contient du $\mathrm{KNO}_{3}$ à $0,2 \mathrm{mmol} / \mathrm{l}$ (fig 6 ), la cinétique d'apparition des nodules n'est pas sensiblement modifiée par rapport au milieu sans azote minéral si les plantes sont inoculées à l'âge de $0 \mathrm{j}(T(I)=0 \mathrm{j})$ et de $15 \mathrm{j}$ $(T(I)=15 \mathrm{j})$. Par contre, lorsque les plantes sont inoculées à l'âge de $30 \mathrm{j}$, lapport de $\mathrm{KNO}_{3}$ devient bénéfique et permet la formation d'un nombre de nodules comparable à celui obtenu lors d'inoculations plus précoces. Toutefois, lorsque les plantes sont inoculées à l'âge de $45 \mathrm{j}(T(I)$ $=45 \mathrm{j}$ ), l'addition de $\mathrm{KNO}_{3}$ n'est plus suffisante pour obtenir une nodulation optimale, malgré son effet encore positif par rapport au milieu dépourvu de $\mathrm{KNO}_{3}$.

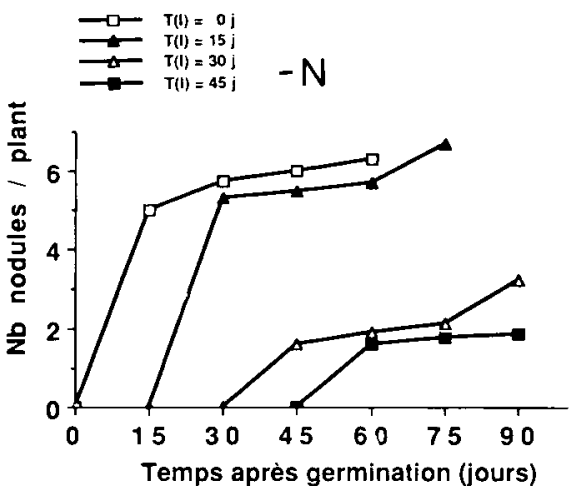

Flg 5. Cinétique de la formation des nodules selon l'âge de la plante au moment de l'inoculation et en l'absence d'azote combiné dans le milieu de culture $(-N)$.
D'ailleurs, les plantes inoculées à l'âge de 45 j restent dans leur majorité chlorosées et peu développées et environ la moitié des plantes meurent après l'inoculation.

\section{Nodulation, ARA et croissance des plantes après 5 mois de croissance}

Après 5 mois de croissance, les plantes des différents traitements ont été prélevées pour évaluer leur croissance et leur $A R A$ en relation avec la nodulation. D'après le tableau I, les différents paramètres étudiés varient dans le même sens quelles que soient les conditions de culture testées : les poids de matière sèche des tiges feuillées et des nodules sont significativement corrélés $(r=0,92)$, le rapport poids de matière sèche des tiges feuillées/ poids de matière sèche des nodules étant proche de 10. L'analyse factorielle de ces données (résultats non décrits) montre que l'effet du facteur principal "période d'inoculation $T(I)$ " est significatif $(P=0,05)$, contrairement au facteur principal «apport de $N$ ", quels que soient les paramètres considérés, tandis que l'interaction des 2 facteurs est significative.

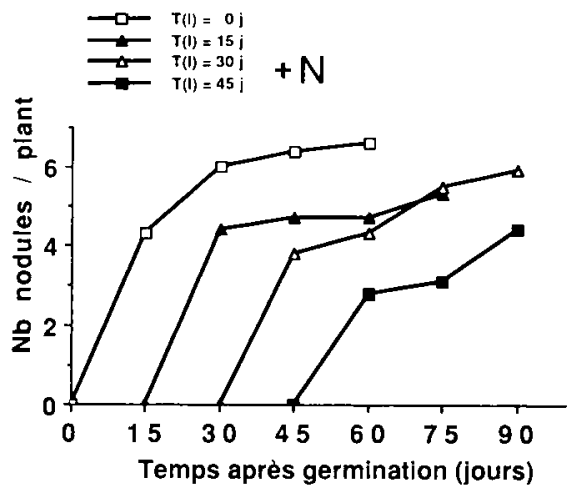

Fig 6. Cinétique de la formation des nodules selon l'âge de la plante au moment de l'inoculation et en présence de $\mathrm{KNO}_{3} 0,2 \mathrm{mmol} / /$ dans le milieu de culture $(+N)$. 
Tableau l. Effets de la date d'inoculation et de l'apport d'une source d'azote minéral sur la croissance, la nodulation et l'activité réductrice d'acétylène d'Acacia mangium après 5 mois de culture.

\begin{tabular}{|c|c|c|c|c|c|}
\hline $\begin{array}{l}\text { Age de la plante } \\
\text { au moment de } \\
\text { l'inoculation }\end{array}$ & $\begin{array}{l}1 \text { Milieu. } \\
\text { minéral }\end{array}$ & $\begin{array}{l}\text { Matière sèche } \\
\text { de tiges feuillées } \\
\text { (mg/plante) }\end{array}$ & $\begin{array}{l}\text { Matière sèche } \\
\text { de nodules } \\
\text { (mg/plante) }\end{array}$ & $\begin{array}{l}\text { Nombre de } \\
\text { nodules } \\
\text { (par plante) }\end{array}$ & $\begin{array}{c}2 \text { ARA } \\
\text { (nmol } C_{2} H_{4} \\
\text { h/plante) }\end{array}$ \\
\hline$T(I)=0 \mathrm{j}$ & $\begin{array}{l}-N \\
+N\end{array}$ & $\begin{array}{l}65 \\
3 \mathrm{a} \\
65\end{array}$ & $\begin{array}{c}6,7 \\
a \\
6,5\end{array}$ & $\begin{array}{c}15,0 \\
a \\
14,4\end{array}$ & $\begin{array}{c}134 \\
a \\
83\end{array}$ \\
\hline$T(l)=15 \mathrm{j}$ & $\begin{array}{l}-\mathrm{N} \\
+\mathrm{N}\end{array}$ & $\begin{array}{l}69 \\
a \\
47\end{array}$ & $\begin{array}{l}6,5 \\
a \\
4,3\end{array}$ & $\begin{array}{l}17,9 \\
a \\
8,9\end{array}$ & $\begin{array}{c}131 \\
a \\
94\end{array}$ \\
\hline$T(l)=30 \mathrm{j}$ & $\begin{array}{l}-\mathrm{N} \\
+\mathrm{N}\end{array}$ & $\begin{array}{l}29 \\
b \\
41\end{array}$ & $\begin{array}{l}2,5 \\
b \\
4,0\end{array}$ & $\begin{array}{l}5,6 \\
b \\
8,3\end{array}$ & $\begin{array}{c}73 \\
a \\
88\end{array}$ \\
\hline$T(l)=45 \mathrm{j}$ & $\begin{array}{l}-\mathrm{N} \\
+\mathrm{N}\end{array}$ & $\begin{array}{l}19 \\
b \\
39\end{array}$ & $\begin{array}{c}0,8 \\
b \\
2,9\end{array}$ & $\begin{array}{c}4,0 \\
b \\
4,1\end{array}$ & $\begin{array}{l}25 \\
b \\
56\end{array}$ \\
\hline
\end{tabular}

${ }^{1}-\mathrm{N}$ : milieu minéral sans source d'azote; $+\mathrm{N}$ : milieu minéral avec $\mathrm{KNO}_{3}\left(0,2 \mathrm{mmol} \vdash^{-1}\right){ }^{2}$ ARA : activité réductrice d'acétylène exprimée en nmol $\mathrm{C}_{2} \mathrm{H}_{4}$ /h/plante. ${ }^{3}$ Les valeurs affectées de la même lettre à l'intérieur de chaque colonne ne sont pas significativement différentes à $P=0,05$, d'après le test de Newman-Keuls $(n=10)$. Pour chaque paramètre étudié, chaque lettre correspond à la moyenne (non reportée sur le tableau) des traitements $-\mathrm{N}$ et $+\mathrm{N}$ mesurée pour chaque date d'inoculation $T(I)$, sachant que le facteur "apport de N" n'a pas d'effet significatif sur la variance.

\section{DISCUSSION ET CONCLUSIONS}

Le dispositif de culture présenté ici, possède plusieurs avantages pour étudier les premières étapes de la nodulation les Légumineuses. Il est simple, peu encombrant et les fibres de polypropylène constituent un support inerte, d'utilisation aisée. Par rapport aux systèmes classiques utilisant des milieux gélosés, il assure une aération et une humidité satisfaisantes au niveau des racines, qui favorisent ainsi les échanges gazeux, notamment l'accessibilité des nodules à l'azote atmosphérique. Avec ce dispositif, les plantes peuvent croître à l'intérieur des tubes en asepsie totale avec un contrôle optimal des différents facteurs (milieu nutritif, conditions de cul- ture...), indépendamment des variations environnementales. II permet de réaliser des observations continues et non destructives de la nodulation. L'analyse de l'activité réductrice d'acétylène pendant les 3 premiers mois (106 j) a montré que les nodules avaient une activité fixatrice d'azote continue dans ce système de culture. L'analyse des différentes parties de la plante est aisée car l'appareil racinaire peut être séparé facilement du substrat. La comparaison des résultats obtenus à 3,5 mois (fig 3) avec ceux obtenus à 5 mois relatifs à $T(I)=0 \mathrm{j}(-\mathrm{N})$ (tableau $\mathrm{I})$, montre que le nombre et le poids de matière sèche de nodules ainsi que-le poids de matière sèche de tiges feuillées (résultats non décrits à 3,5 mois) continuent à aug- 
menter entre ces 2 dates tandis que l'ARA se maintient au même niveau avec une moyenne proche de $120 \mathrm{nmol} \cdot \mathrm{h}^{-1}\left(\mathrm{C}_{2} \mathrm{H}_{4}\right) /$ plante dans les 2 cas. Ces résultats suggèrent que le dispositif assure un développement satisfaisant de la plante pendant au moins 5 mois, bien que l'éclairement ambiant soit limitant en chambre de culture et réduise l'activité photosynthétique des plantes.

Dans nos conditions expérimentales, les nodules racinaires apparaissent au niveau des points d'émergence des racines secondaires à proximité de la racine principale. Pour la majorité des espèces de Légumineuses étudiées, l'infection a lieu au niveau de la zone de croissance des poils absorbants, les poils immatures encore peu développés constituant la voie principale de pénétration des rhizobiums. II en est ainsi chez Glycine max (Bhuvaneswari et al, 1980; Turgeon et Bauer, 1982), Vigna sinensis, Medicago sativa (Bhuvaneswari et al, 1981), Macroptilium atropurpureum (Ridge et Rolfe, 1986). Chez $A$ mangium, nous n'avons pas observé de relation entre la distribution des nodules et la localisation des poils absorbants (résultats non présentés ici). Le développement des nodules sur la partie basale des racines secondaires pourrait faire penser à un phénomène de pénétration intercellulaire de type crack entry décrit chez d'autres Légumineuses comme Arachis hypogea (Chandler, 1978), Sesbania rostrata (Tsien et al, 1983; Duhoux, 1984), Stylosanthes sp (Ranga Rao, 1977), Aeschynomene afraspera (Alazard et Duhoux, 1990). L'analyse cytologique des premières étapes de linfection, que nous avons entreprise, devrait nous permettre de préciser le mode d'infection des racines d'A mangium par les Bradyrhizobium.

Une caractéristique importante de la nodulation est la période d'apparition des nodules. Notre dispositif expérimental a per- mis de mettre en évidence 3 vagues successives de formation de nodules au cours du temps. Cette rythmicité pourrait résulter d'un phénomène d'autorégulation de la nodulation, phénomène décrit chez quelques Légumineuses (Pierce et Bauer, 1983; Malik et Bauer, 1988). La rythmicité observée concerne uniquement le nombre de nodules formés et non pas les autres paramètres : poids de matière sèche des tiges feuillées, périodes d'apparition des feuilles (résultats non présentés ici), poids de matière sèche des nodules et $A R A$, qui augmentent de manière continue au cours du temps. Chez le soja, étudié au champ, Gibson et al (1982) ont décrit également une augmentation rythmique du poids de matière sèche des nodules et de leur activité réductrice d'acétylène spécifique (SARA); mais les rythmes décrits pouvaient être attribués soit à des facteurs environnementaux, soit à d'éventuels facteurs endogènes de variation. $\mathrm{Au}$ contraire, avec notre dispositif qui assure le contrôle des conditions de culture et une observation continue du système racinaire, la rythmicité de la nodulation observée ne peut être attribuée qu'à l'action de facteurs endogènes. Notre dispositif pourra être utilisé ultérieurement pour préciser l'origine de la formation rythmique des nodules d' $A$ mangium.

La date optimale d'inoculation après la germination des plantes a été déterminée. L'ARA et la croissance des plantes n'ont pas été modifiées lorsque l'inoculation a eu lieu 0 ou $15 \mathrm{j}$ après la germination, que le milieu nutritif contienne ou non de l'azote. En l'absence d'azote combiné, lorsque l'inoculation a eu lieu 30 ou 45 j après la germination, la nodulation, l'ARA et la croissance des plantes étaient réduites. La faible nodulation observée s'explique par un mauvais état physiologique de la plante résultant de la carence en azote combiné. Par contre, en présence d'azote, la nodula- 
tion et la croissance des plantes inoculées sont satisfaisantes tant que l'inoculation a eu lieu avant $45 \mathrm{j}$. Ces résultats confirment l'effet favorable de la présence d'une faible dose d'azote (azote starter) dans le milieu de culture sur la nodulation.

Après 5 mois de culture dans ce dispositif, les plantes ont une croissance et une activité fixatrice d'azote corrélées à leur nombre de nodules (tableau I). Or, le nombre de nodules formés sur ces mêmes plantes variait déjà dans le même sens 15 j après leur inoculation (fig 5). Ces observations suggèrent que des tests de sélection précoce peuvent être mis en place pour identifier les couples performants de la symbiose.

Le dispositif décrit ici nous a permis d'optimiser les conditions de culture in vitro de l'Acacia mangium. Il a été utilisé au laboratoire avec d'autres Légumineuses et certaines plantes actinorhiziennes. II s'est révélé particulièrement efficace pour tester l'effectivité de souches variées de Bradyrhizobium sp chez $A$ mangium, en accord avec les résultats obtenus au champ (résultats non publiés). Ce dispositif pourrait être également employé pour sélectionner de façon précoce des plantes hôtes à haut potentiel fixateur d'azote.

\section{REMERCIEMENTS}

Nous remercions YR Dommergues pour ses conseils lors de la préparation du manuscrit.

\section{RÉFÉRENCES}

Alazard D, Duhoux E (1990) Development of stem nodules in a tropical forage legume, Aeschynomene afraspera. J Exp Bot 41, 1199-1206
Anonyme (1983) Fichier technique de la fixation symbiotique de l'azote Légumineusel Rhizobium. FAO, Rome

Bhuvaneswari TV, Turgeon BG, Bauer WD (1980) Early events in the infection of soybean (Glycine max L Merr) by Rhizobium japonicum. I. Localization of infectible root cells. Plant Physiol 66, 1027-1031

Bhuvaneswari TV, Bhagwat AA, Bauer WD (1981) Transient susceptibility of root cells in four common legumes to nodulation by rhizobia. Plant Physio/ 68, 1144-1149

Chandler MR (1978) Some observations on infection of Arachis hypogea $L$ by Rhizobium. $J$ Exp Bot 29, 747-755

Dart PJ (1977) Infection and development of leguminous nodules. In : $A$ treatise on dinitrogen fixation. Section 3. Biology (RWF Hardy, WS Silver, eds) John Wiley and Sons, New York, 367-472

Dhawan V, Bhojwani SS (1987) In vitro nodulation of micropropagated plants of Leucaena leucocephala by Rhizobium. Plant Soil 103 , 274-276

Duhoux E (1984) Ontogenèse des nodules caulinaires de Sesbania rostrata (Légumineuses). Can J Bot 62, 982-994

Galiana A, Diem HG, Dommergues $Y$ (1990) Nitrogen fixation potential of Acacia mangium and Acacia auriculiformis seedlings inoculated with Bradyrhizobium and Rhizobium spp. Biol Fertil Soils 9, 261-267

Gibson AH, Dreyfus BL, Dommergues YR (1982) Nitrogen fixation by legumes in the tropics. In : Microbiology of tropical soils and plant productivity (YR Dommergues, HG Diem, eds) Martinus Nijhoff/Dr W Junk publishers, The Hague, 37-73

Hardy RW, Holsten RD, Jackson EK, Burns RC (1968) The acetylene ethylene assay for $\mathrm{N}_{2}$ fixation: laboratory and field estimation. Plant Physiol 43, 1185-1207

Lakshminarayana K, Narula N, Tauro P (1988) A simple technique to obtain nodulation of chickpea and some large-seeded legumes in agar tubes. Trop Agric (Trinidad) 65, 121-124

Malik NSA, Bauer WD (1988) When does the self-regulatory response elicited in soybean root after inoculation occur ? Plant Physiol 88, 537-539 
Natl Acad Sci USA (1983) In: Mangium and other fast-growing acacias for the humid tropics. US Natl Acad Press, Washington, DC, 18-19

NFT Highlights (1987) Acacia mangium - A fast-growing tree for the humid tropics. Nitrogen Fixing Tree Assoc, 87-04, July 1987

Pierce M, Bauer WD (1983) A rapid regulatory response governing nodulation in soybean. Plant Physiol 73, 286-290

Pomier M, Beligne V, Bonneau X, de Taffin G (1986) Restauration de la fertilité des sols lors de la replantation d'une cocoteraie. Oléagineux 41, 223-228

Ranga Rao V (1977) Effect of root temperature on the infection processes and nodulation in Lotus and Stylosanthes. J Exp Bot 28, 241259

Ridge RW, Rolfe BG (1986) Sequence of events during the infection of the tropical legume Macroptilium atropurpureum UrB by the broad-host-range, fast-growing Rhizobium ANU240. J Plant Physiol 122, 121-137
Rigaud J (1981) Comparison of the efficiency of nitrate and nitrogen fixation in crop yield. $I n$ : Nitrogen and carbon metabolism (JD Bewley, ed) Martinus Nijhoff Publishers, The Hague, 17-48

Takats ST (1986) Suppression of nodulation in soybeans by superoptimal inoculation with Bradyrhizobium japonicum. Physiol Plant 66, 669-673

Tsien HC, Dreyfus BL, Schmidt EL (1983) Initial stages in the morphogenesis of nitrogenfixing stem nodules of Sesbania rostrata. J Bacteriol 156, 888-897

Turgeon BG, Bauer WD (1982) Early events in the infection of soybean by Rhizobium japonicum. Time course and cytology of the initial infection process. Can J Bot 60, 152-161

Vincent JM (1970) A manual for the practical study of root nodule bacteria. International Biological Programme Handbook no 15. Blackwell Sci Publ, Oxford 